\title{
ARCHITECT HIV Combo Ag/Ab and RealTime HIV-1 Assays Detect Diverse HIV Strains in Clinical Specimens
}

\author{
Mary A. Rodgers,, Ana S. Vallari, Julie Yamaguchi,, Vera Holzmayer,, Barbara Harris, \\ Coumba Toure-Kane, Souleymane Mboup, Samar Badreddine, ${ }^{3}$ Carole McArthur, ${ }^{4,5}$ \\ Nicaise Ndembi, Dora Mbanya, Lazare Kaptue, and Gavin Cloherty ${ }^{1}$
}

\begin{abstract}
Periodic evaluation of the impact of viral diversity on diagnostic tests is critical to ensure current technologies are keeping pace with viral evolution. To determine whether HIV diversity impacts the ARCHITECT HIV Combo Ag/ Ab (HIV Combo) or RealTime HIV-1 (RT) assays, a set of $N=199$ HIV clinical specimens from Cameroon, Senegal, Saudi Arabia, and Thailand were sequenced and tested in both assays. The panel included historical groups $\mathrm{N}$ and $\mathrm{P}$ specimens and a newly identified group $\mathrm{N}$ specimen. These and specimens classified as $\mathrm{H}, \mathrm{U}$ (unclassified)/ URF (unique recombinant form), CRF (circulating recombinant form) 01, 02, 06, 09, 11, 13, 18, 22, 37, and 43 were detected by both the RT assay (1.75-6.84 log copies/ml) and the HIV Combo assay (3.26-1121.96 sample to cutoff ratios). Sequence alignment identified 3 or fewer mismatches to the RT assay oligos in $82.4 \%$ of samples. Altogether, these data demonstrate the HIV Combo and RT assays detect diverse strains of HIV in clinical specimens.
\end{abstract}

Keywords: HIV diversity, diagnostics, serology, nucleic acid test

T HE DEEP GENETIC DIVERSITY of HIV-1 is primarily driven by an error-prone viral reverse transcriptase, high rates of recombination, and multiple cross-species transmission events. ${ }^{1}$ To date, 4 groups of HIV-1 (M, N, O, and P) have been identified, as well as 9 major subtypes $(\mathrm{A}, \mathrm{B}, \mathrm{C}, \mathrm{D}$, $\mathrm{F}, \mathrm{G}, \mathrm{H}, \mathrm{J}$, and $\mathrm{K})$, and at least 90 circulating recombinant forms (CRFs) of group M (Los Alamos National Laboratories HIV database, date of accession: September 8, 2017). Local pandemics in some parts of the world are limited to 1 or 2 major strains of HIV, while regions of sub-Saharan Africa have reported all major strains in circulation. ${ }^{1}$ However, with increases in global travel, immigration, and military deployment, regions outside of sub-Saharan Africa are experiencing increases in HIV-1 strain diversity. Sequence comparisons indicate that the average nucleotide divergence between HIV1 groups is $37.5 \%$, with $14.7 \%$ between subtypes, and $8.2 \%$ within subtypes. ${ }^{2}$ This extensive sequence heterogeneity is a challenge for the design of therapeutics, vaccines, blood screening, and diagnostic tests, which rely fundamentally on sequence conservation.

The detection of diverse strains by the RealTime HIV-1 assay (RT; Abbott Molecular Diagnostics, Des Plaines, IL) and the HIV Combo Ag/Ab assay (HIV Combo; Abbott Diagnostics, Abbott Park, IL) reported in several recent publications largely relied on cultured virus isolates. ${ }^{3-9}$ In contrast to cultured virus isolates, clinical specimens are complex sample types that are representative of the samples tested in clinical diagnostic laboratories. Therefore, evaluation of the detection of diverse HIV-1 strains in collections of unique clinical specimens is an important challenge to determine whether sequence and specimen diversity may affect assay performance. For both the HIV Combo and RT assays,

\footnotetext{
${ }^{1}$ Infectious Disease Research, Abbott Diagnostics, Abbott Park, Illinois.

${ }^{2}$ Institut de Recherche en Santé, de Surveillance Epidemiologique et de Formations, Dakar, Senegal.

${ }^{3}$ Infection Control Unit, King Faisal Specialist Hospital and Research Center, Jeddah, Saudi Arabia.

${ }_{5}^{4}$ Department of Oral and Craniofacial Sciences, School of Dentistry, University of Missouri-Kansas City, Kansas City, Missouri.

${ }^{5}$ Pathology Department, Truman Medical Center, Kansas City, Missouri.

${ }^{6}$ Laboratory Research Department, Institute of Human Virology, Abuja, Nigeria.

${ }^{7}$ Department of Hematology, Université de Yaoundé I, Yaoundé, Cameroon.

${ }^{8}$ Laboratoire D’Hematologie, Université des Montagnes, Bangangté, Cameroon.
}

(C) Mary A. Rodgers et al. 2018; Published by Mary Ann Liebert, Inc. This Open Access article is distributed under the terms of the Creative Commons License (http://creativecommons.org/licenses/by/4.0), which permits unrestricted use, distribution, and reproduction in any medium, provided the original work is properly cited. 

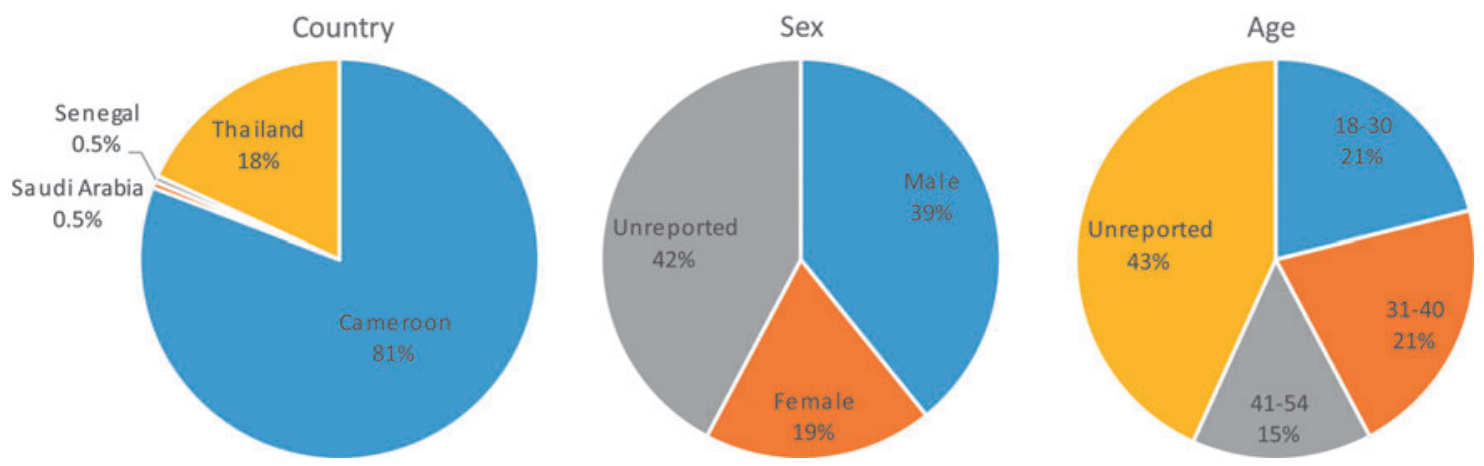

FIG. 1. Summaries of demographics. The country, sex, and age at the time of specimen collection are plotted in pie charts as percentages of the total number of samples $(N=199)$. The full age range of the study cohort is $18-54$ years with a median age of 34 years.

antigen-only cultured virus isolates have been used to demonstrate the detection of rare $\mathrm{CRF}$, groups $\mathrm{N}, \mathrm{O}$, and $\mathrm{P}$ specimens, ${ }^{4,5}$ and clinical specimens have been tested for up to 13 different CRFs, group $\mathrm{O}$, group $\mathrm{P}$, and rare subtypes $\mathrm{H}$, $\mathrm{J}$, and $\mathrm{K}^{3,6-8,10}$ However, clinical specimens for newly identified group $\mathrm{N}$, group $\mathrm{P}$, and CRF specimens must also be tested as these branches of the HIV phylogenetic tree continue to diversify. Toward this end, we have challenged the HIV Combo and RT assays with a diverse set of previously untested clinical HIV-1 specimens, including rare CRFs, group $\mathrm{N}$, and group $\mathrm{P}$ specimens.

Specimens $(N=199)$ were collected by the Abbott Global Surveillance Program through collaborations in Cameroon, Saudi Arabia, Senegal, and Thailand (Fig. 1). All specimens were obtained according to local regulations in each country at the time of collection between 1998 and 2016, including local IRB approval when required. Specimens were identified as HIV-1 positive by rapid diagnostic tests done in source countries before sequence analysis and testing at Abbott Laboratories. Age and gender demographic information were available for the majority of participants, of which $67 \%$ were male and the median age was 34 years with an interquartile range of 23 (Fig. 1).

Specimens were sequenced as previously described in the polymerase integrase ( $p o l \mathrm{IN}$ ) region and when specimen volume allowed, the env IDR region was also sequenced. ${ }^{8}$ Complete genome or subgenomic sequences for 14 rare specimens included in this study have been previously reported: group $\mathrm{P}$ specimen $\mathrm{U} 14788,{ }^{11}$ group $\mathrm{N}$ specimens $\mathrm{S} 4858,{ }^{7} \mathrm{U} 14296,{ }^{12}$ and DJO0135, ${ }^{13}$ subtype H specimen $\mathrm{K} 562,{ }^{7}$ subtype $\mathrm{G}$ specimen $\mathrm{J} 11254,{ }^{14}$ and eight CRF specimens. ${ }^{7}$ Viral load data had not been collected for any of these samples when they were originally sequenced and only S4858, K562, and the CRF specimens have been previously tested by HIV Combo. ${ }^{7}$ The pol IN and env IDR sequences from these samples were included in the subsequent phylogenetic analysis to confirm subgenomic classifications with an updated reference sequence alignment before testing by the HIV Combo and RT assays.

The pol IN and env IDR sequences were classified by phylogenetic inference with reference sequences obtained from the Los Alamos National Laboratories database for SIVcpz, SIVgor, group N, group $\mathrm{P}$, group $\mathrm{O}$, group $\mathrm{M}$ subtypes A-K, and CRFs 1-90 as previously described. ${ }^{7}$ After removing the $\mathrm{CRF}$ references that did not branch with or near the specimen sequences, a final phylogenetic tree was built for each region sequenced as shown in Figure 2, and the classifications are summarized in Table 1. For specimens with different classifications in the two sequenced regions, the final classification of URF (unique recombinant form) was assigned. Notably, specimen J11254 was reclassified as CRF43 as it clearly branched with CRF43 reference sequences and one new group $\mathrm{N}$ specimen was identified, U22816, which is the 17th sequence confirmed group $\mathrm{N}$ infection reported to date (Fig. 3). U22816 was collected in 2012 from a 48-year-old female in Yaoundé, Cameroon, and it is basal to other group $\mathrm{N}$ sequences in both the pol IN and env IDR regions (Fig. 2).

Since the HIV Combo assay targets a region of the env IDR and the RT assay targets a region of the pol IN, the classifications of these individual regions were used for challenging each respective assay. Notably, many specimens had sequences that did not branch with any references or were very basal to reference sequences (Fig. 2). These sequences were called unclassified (U) and recombinant analysis using Simplot software identified unique breakpoints, indicating they might be URFs.

All 199 samples were tested by the RT assay according to the manufacturer's protocol. The majority of specimens were tested neat, although specimens with limited volume were diluted 1:10 in HIV-negative human plasma before testing. The reported viral load (log copies $/ \mathrm{ml})$ values were adjusted to account for dilution factors. The $\log$ copies $/ \mathrm{ml}$ result ranges and averages for each HIV pol IN classification are shown in Figure 3. All specimens were detected regardless of classification with a viral load ranging from 1.75 to $6.84 \log _{10}$ copies/ $\mathrm{ml}$ (Fig. 3). When the pol IN sequences were compared to the forward primer, probe, and reverse primer in the RT assay, the number of combined mismatches for all three regions ranged from 0 to 11 (Fig. 3), with the most mismatches in the group $\mathrm{P}$ sequence. The majority of specimens $(N=164,82.4 \%)$ had three total mismatches or less, indicating that the region for the RT amplicon is well conserved. Importantly, all the samples were detected despite the identified primer and probe mismatches, indicating that the RT assay can accommodate the full breadth of existing HIV diversity.

Specimens were also tested in the HIV Combo assay. While most were tested neat, the rare groups $\mathrm{N}$ and $\mathrm{P}$ samples were diluted 1:10-1:50 and S/CO results were not 

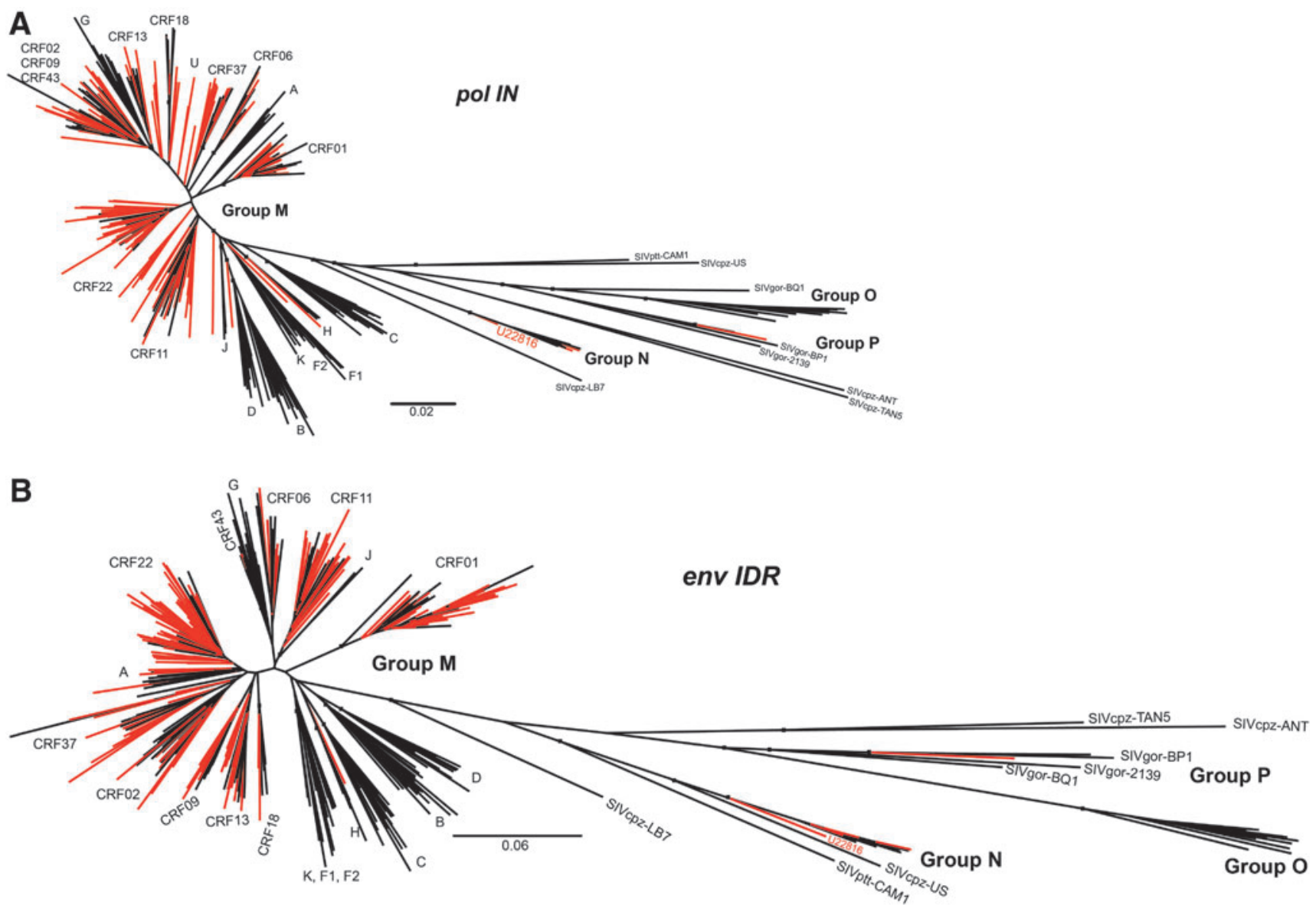

FIG. 2. Phylogenetic trees. The phylogenetic trees for the pol IN (1009nt, A) and env IDR (676nt, B) are shown with specimen sequences indicated in red and reference sequences indicated in black. Relevant nodes with bootstrap values $>70$ are labeled by small black boxes. CRF, circulating recombinant form; U, unclassified.

Table 1. Summary of Classifications

\begin{tabular}{lrrr}
\hline Classification & pol IN & env IDR & Overall \\
\hline CRF01 & 36 & 36 & 36 \\
CRF02 & 26 & 11 & 11 \\
CRF06 & 4 & 9 & 4 \\
CRF09 & 6 & 6 & 6 \\
CRF11 & 24 & 25 & 18 \\
CRF13 & 5 & 14 & 4 \\
CRF18 & 3 & 3 & 3 \\
CRF22 & 56 & 57 & 51 \\
CRF37 & 9 & 15 & 8 \\
CRF43 & 1 & 1 & 1 \\
U/URF & 23 & 7 & 51 \\
H & 1 & 1 & 1 \\
A & 0 & 4 & 0 \\
Not sequenced & NA & 5 & NA \\
Group N & 4 & 4 & 4 \\
Group P & 1 & 1 & 1 \\
Total & 199 & 199 & 199 \\
\hline
\end{tabular}

The classifications determined by phylogenetic inference as shown for the pol IN and env IDR sequences. Overall classifications were determined by comparing pol IN and env IDR individual classifications. Where regions did not match, an overall classification of URF was made. $N=5$ specimens could not be sequenced in the env IDR region. The overall classifications for these samples were determined solely by the pol IN sequence.

CRF, circulating recombinant form; NA, not applicable; U, unclassified; URF, unique recombinant form. adjusted for dilution since the assay is not quantitative. All specimens were reactive in the HIV Combo assay, with a range of 3.26-1121.96 S/CO (Fig. 3), indicating that this assay can also detect diverse HIV specimens. As expected, specimens that were tested in both assays had $100 \%$ concordant results.

In this study, new group $\mathrm{N}$, subtype $\mathrm{H}, \mathrm{U} / \mathrm{URF}$, and CRF01, 02, 06, 09, 11, 13, 18, 22, 37, and 43 sequences are reported that further expand the known genetic diversity of HIV-1. The RT and HIV Combo assays successfully detected these geographically and genetically diverse HIV-positive clinical specimens. Previous studies that challenged RT and HIV Combo assay performance with diverse strains of HIV relied on virus isolate cultures for some rare strains, such as groups $\mathrm{N}$ and $\mathrm{P}^{4,5,15}$ In contrast to native specimens, virus culture isolates do not contain HIV-1 antibodies and therefore do not completely represent the complexity of human specimens. The results of this study indicate that both assays can detect diverse strains of HIV-1, accommodating both sequence and geographical diversity. Both assays were developed before groups $\mathrm{N}$ and $\mathrm{P}$ strains were identified and can readily detect these new strains without any further modification to the assays, which suggests that these assays will continue to be able to detect newly emerging strains of HIV as they arise. As HIV-1 diversity continues to present new challenges for diagnostic assays, viral surveillance remains 
FIG. 3. Detection of HIV infection in diverse clinical specimens. The range and mean viral load (A) and S/CO (B) detected from clinical specimens of each HIV classification are plotted. The classifications shown in (A) are for the pol IN region and the classifications indicated in (B) are for the env IDR region. The range and mean number of total mismatches in the HIV RealTime primers and probe are plotted for each pol IN classification in (C). Only one clinical specimen was available for CRF43, $\mathrm{H}$, and $\mathrm{P}$. URF, unique recombinant form.
A 1000

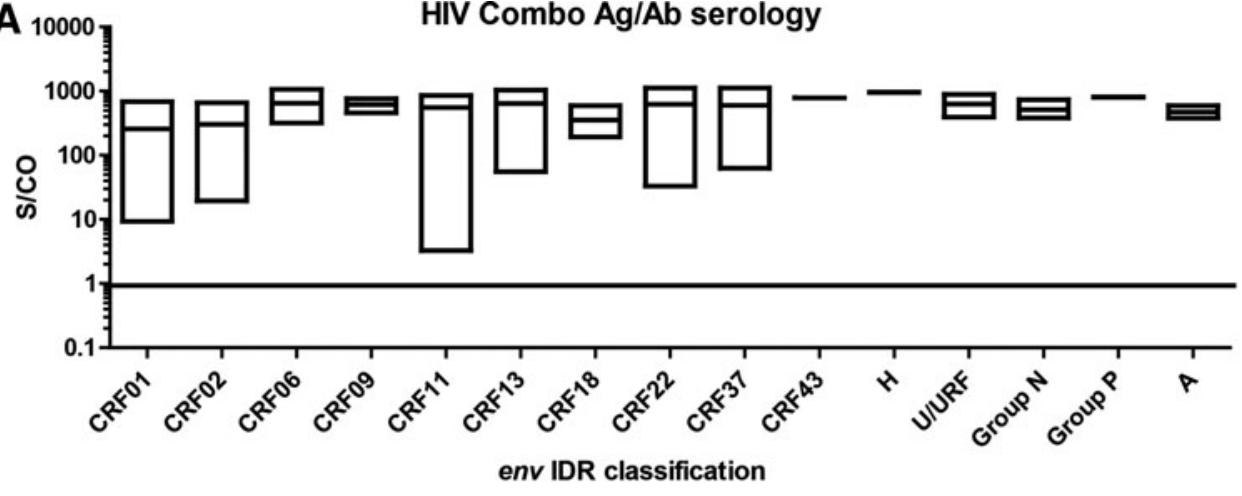

B

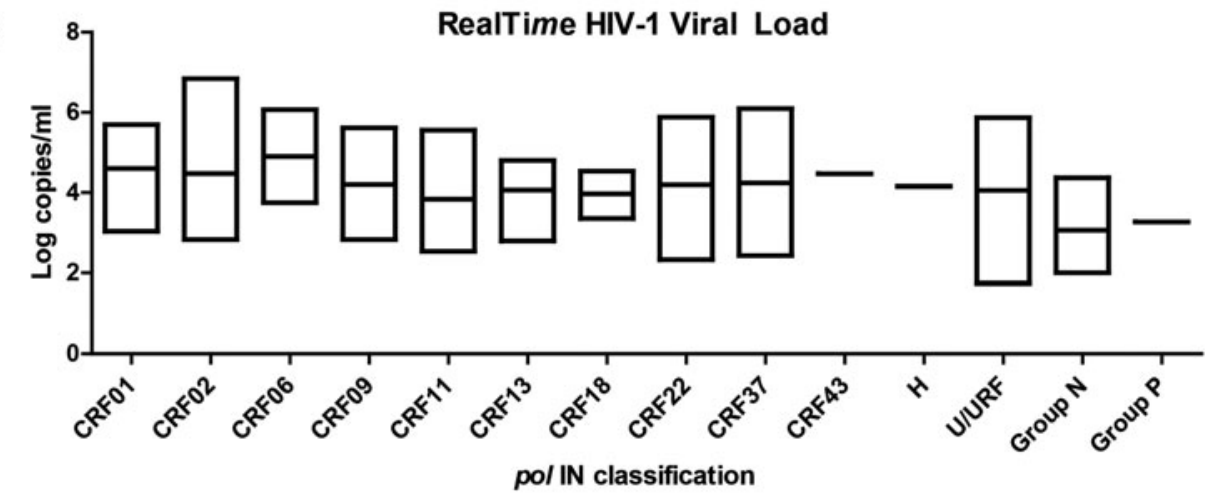

C

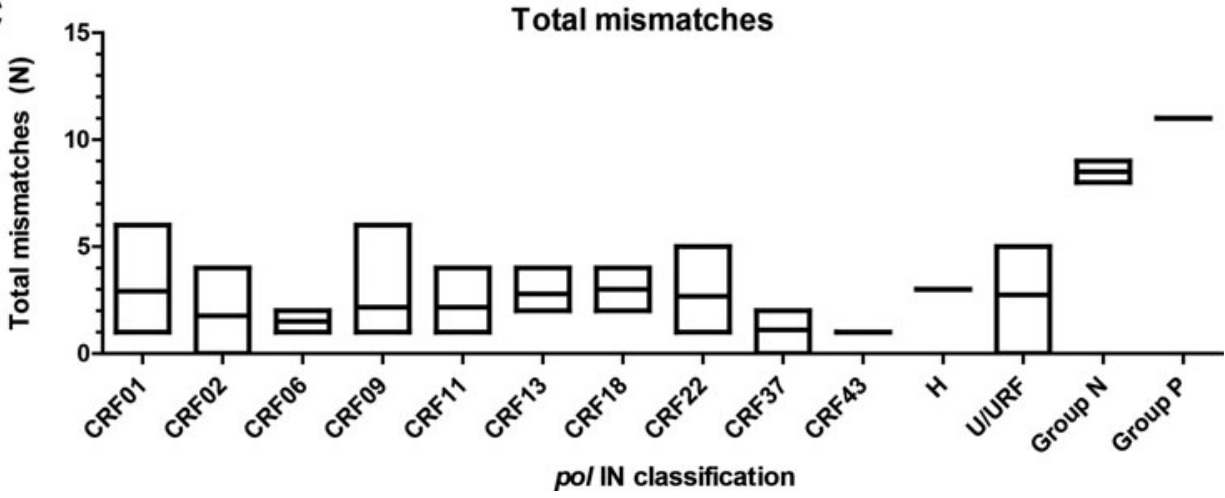

essential for identification of specimens from divergent strains of HIV-1 to ensure diagnostic tests continue to accurately detect infections regardless of where they are acquired.

\section{Sequence Data}

The polymerase sequences have been deposited in Genbank under accession numbers MG012032-MG012216 and envelope sequences have been deposited under accession numbers MG022442-MG022621.

\section{Acknowledgments}

The authors thank the National Blood Centre of the Thai Red Cross Society in Bangkok, Thailand, for providing specimens for this study and Jill Fuhrman for laboratory support.

\section{Author Disclosure Statement}

This study was funded by Abbott Laboratories.

\section{References}

1. Lihana RW, Ssemwanga D, Abimiku A, Ndembi N: Update on HIV-1 diversity in Africa: A decade in review. AIDS Rev 2012;14:83-100.

2. Li G, Piampongsant S, Faria NR, et al.: An integrated map of HIV genome-wide variation from a population perspective. Retrovirology 2015;12:18.

3. Berg MG, Yamaguchi J, Alessandri-Gradt E, Tell RW, Plantier JC, Brennan CA: A Pan-Hiv strategy for complete genome sequencing. J Clin Microbiol 2015;54:868-882.

4. Swanson P, Huang S, Abravaya K, et al.: Evaluation of performance across the dynamic range of the Abbott RealTime HIV-1 assay as compared to VERSANT HIV-1 RNA 3.0 and AMPLICOR HIV-1 MONITOR v1.5 using serial dilutions of 39 group $\mathrm{M}$ and $\mathrm{O}$ viruses. J Virol Methods 2007;141:49-57.

5. Qiu X, Sokoll L, Yip P, et al.: Comparative evaluation of three FDA-approved HIV Ag/Ab combination tests using a genetically diverse HIV panel and diagnostic specimens. J Clin Virol 2017;92:62-68. 
6. Luk K-C, Berg MG, Naccache SN, Kabre B, Federman S, Mbanya D, Kaptue L, ChiuCY, Brennan CA, Hackett J Jr: Utility of metagenomic next-generation sequencing for characterization of HIV and human pegivirus diversity. PLoS One 2015;10:e0141723.

7. Rodgers MA, Vallari AS, Harris B, et al:: Identification of rare HIV-1 group N, HBV AE, and HTLV-3 strains in rural South Cameroon. Virology 2017;504:141-151.

8. Rodgers MA, Wilkinson E, Vallari A, et al.: Sensitive nextgeneration sequencing method reveals deep genetic diversity of HIV-1 in the democratic republic of the congo. J Virol 2017;91:e01841-16.

9. Tang N, Huang S, Salituro J, et al.: A RealTime HIV-1 viral load assay for automated quantitation of HIV-1 RNA in genetically diverse group $\mathrm{M}$ subtypes $\mathrm{A}-\mathrm{H}$, group $\mathrm{O}$ and group N samples. J Virol Methods 2007;146:236-245.

10. Plantier JC, Leoz M, Dickerson JE, et al.: A new human immunodeficiency virus derived from gorillas. Nat Med 2009; $15: 871-872$.

11. Vallari A, Holzmayer V, Harris B, et al.: Confirmation of putative HIV-1 group P in Cameroon. J Virol 2011;85: 1403-1407.

12. Vallari A, Bodelle P, Ngansop C, et al.: Four new HIV-1 group $\mathrm{N}$ isolates from Cameroon: Prevalence continues to be low. AIDS Res Hum Retroviruses 2010;26:109_ 115 .

13. Yamaguchi J, McArthur CP, Vallari A, et al:: HIV-1 Group $\mathrm{N}$ : Evidence of ongoing transmission in Cameroon. AIDS Res Hum Retroviruses 2006;22:453-457.

14. Badreddine $\mathrm{S}$, Smith $\mathrm{K}$, van $\mathrm{Zyl} \mathrm{H}$, et al.: Identification and characterization of HIV type 1 subtypes present in the Kingdom of Saudi Arabia: High level of genetic diversity found. AIDS Res Hum Retroviruses 2007;23:667-674.

15. Mourez T, Delaugerre C, Vray M, Lemee V, Simon F, Plantier JC: Comparison of the bioMerieux NucliSENS EasyQ HIV-1 v2.0-HIV-1 RNA quantification assay versus Abbott RealTime HIV-1 and Roche Cobas TaqMan HIV-1 v2.0 on current epidemic HIV-1 variants. J Clin Virol 2015;71:76-81.

Address correspondence to: Mary A. Rodgers, PhD Infectious Disease Research Abbott Diagnostics 100 Abbott Park Road Abbott Park, IL 60064

E-mail: mary.rodgers@abbott.com 